\title{
A Case of Localized Scleroderma Associated with Osteomyelitis
}

Eiji MUROI, Fumihide OGAWA, Toshifumi YAMAOKA, Fumiko SUEYOSHI, and Shinichi SATO

Department of Dermatology, Nagasaki University Graduate School of Biomedical Sciences, 1-7-1 Sakamoto, Nagasaki 852-8501, Japan.

Phone: +81-95-819-7333

Fax: +81-95-849-7335

E-mail: s-sato@nagasaki-u.ac.jp

Short title: localized scleroderma 


\section{ABSTRACT}

We report a four-year-old girl presented with progressive linear scleroderma affecting the right leg. Biopsy specimen disclosed typical histopathological findings of localized scleroderma. Right leg magnetic resonance image (MRI) showed high signal areas on T2 weighted images on the subcutaneous fatty tissue, muscles, and bone marrow, suggesting that skin inflammation extended to the bone marrow. Oral corticosteroid therapy was instituted with improvement of both skin sclerosis and MRI findings. Our observations suggest that MRI examination should be considered in patents with localized scleroderma to evaluate the extension of the inflammation.

Key words: localized scleroderma, osteomyelitis, MRI, anti-ssDNA antibody, corticosteroid 


\section{INTRODUCTION}

Scleroderma is divided into two clinical entities: localized scleroderma and systemic sclerosis (SSc). Localized scleroderma differs from SSc in that it is not accompanied by Raynaud's phenomenon, acrosclerosis, and internal organ involvement ${ }^{1}$. However, since autoimmune abnormalities of localized scleroderma have been well recognized, this disease has generally been considered to have an autoimmune background ${ }^{2}$. Extensive involvement of the subcutaneous tissue and underlying bony structures is known ${ }^{3}$. Here, we report a patient with localized scleroderma associated with the bone marrow inflammation revealed by the magnetic resonance image (MRI) examination. 


\section{CASE REPORT}

The patient was a four-year-old girl without family history of scleroderma. She had been clinically well until three years ago when she presented with swelling and sclerotic plaques associated with hypo- and hyperpigmentation on the right lower leg, which extended gradually to whole of the right leg. The lesions were not erythematous and did not exhibit lilac ring (Fig 1a, b). Skin abnormalities did not appear on the other parts of body. Raynaud's phenomenon was absent. Mild ankylosis of the right knee and limp were noted. Hematochemical investigations including transaminases, alkaline phosphatase, creatinine, creatine kinase, and blood urea nitrogen were in the normal range. Aldolase levels were elevated to $19.6 \mathrm{IU} / \mathrm{l}$ (normal value, 1.6 to $13.1 \mathrm{IU} / \mathrm{l}$ ) and serum procollagen III peptide (P-III-P) levels were $1.8 \mathrm{U} / \mathrm{l}(<1.0 \mathrm{U} / \mathrm{ml})$. Positive antinuclear antibody (Ab) (1:80, a nucleolar pattern), increased anti-single-stranded deoxyribonucleic acid (ssDNA) Ab (49.6 AU/l; normal value, <25 AU/ml), and increased anticardiolipin $\mathrm{Ab}(20.6 \mathrm{U} / \mathrm{ml} ;<10 \mathrm{U} / \mathrm{ml})$ were observed. Anti-topoisomerase I Ab, anti-RNP Ab, anti-Sm Ab, anti-double-stranded DNA Ab, anti-SS-A Ab, anti-SS-B $\mathrm{Ab}$, and lupus anticoagulant were all negative. Borrelia burgdorferi infection was 
excluded based upon the absence of specific Ab. Skin biopsy showed thickened and increased collagen in the dermis and infiltration of lymphocytes and plasmacytes in the dermis, subcutaneous fatty tissue, and fascia, which confirmed the diagnosis of linear scleroderma (Fig 2a, b). MRI showed the widespread lesion with high signal intensity on T2 weighted images in subcutaneous fatty tissue, vastus intermedius, sartorius muscle, femur, tibia, and bone marrows beneath the skin lesion (Fig 3a, b). Bone destruction was not observed. A bone marrow biopsy specimen from her right ilium showed no neoplastic change. Renal and pulmonary function tests were performed with normal results. Treatment with oral prednisolone of $10 \mathrm{mg}$ daily was instituted with improvement of her skin sclerosis. Then, the patient was treated with a consecutive tapering with oral prednisolone. Serum levels of aldolase, P-III-P, and anti-ssDNA Ab decreased to $6.8 \mathrm{IU} / \mathrm{l}, 1.0 \mathrm{U} / \mathrm{l}$, and $2.6 \mathrm{AU} / \mathrm{ml}$, respectively. MRI scans after eight months showed that the affected area was reduced compared with those before treatment (Fig 3c). 


\section{DISCUSSION}

Localized scleroderma is a connective tissue disorder that is limited to the skin and the

underlying tissue. In localized scleroderma, lesions may extend to and can involve the underlying muscles and bones with disturbances in growth and ankylosis ${ }^{3}$. Recent MRI studies of patients with “en coup de sabre”, a variant of linear scleroderma, have revealed intracranial abnormalities, including multiple lesions with high signal intensity on T2 weighted images, cerebral atrophy, impression of the skull corresponding to the skin lesions ${ }^{4,5}$. In addition, brain histology has shown patchy perivascular and brain lymphocytic infiltration but not necrosis of vessel walls, suggesting that a inflammatory process is involved in the development of lesions ${ }^{4}$. In our case, MRI revealed that T2 signal increase in the right leg extended to the bone marrow directly adjacent to the area of localized scleroderma. Bone marrow biopsy showed no neoplastic change, suggesting that it is an inflammatory lesion. In addition, MRI displayed reduction of the bone marrow lesions in parallel with her improvement of skin lesions by oral corticosteroid therapy. Thus, it is suggested that this osteomyelitis was the result of inflammation extended from skin. 
Localized scleroderma is generally considered to have an autoimmune background because it is accompanied by various immunological abnormalities, such as antinuclear Ab, anti-ssDNA Ab, rheumatoid factor, anti-cardiolipin Ab, and lupus erythematosus cell phenomenon ${ }^{6,7}$. A previous study has shown that anti-cardiolipin Ab is elevated in localized scleroderma with high frequency and correlates with the disease severity $^{7}$. The titer of anti-ssDNA Ab is well correlated with disease activity and responds to oral corticosteroid treatment in patients with severe muscle involvement ${ }^{2}$. In our patient, anti-ssDNA Ab levels were elevated and then decreased in parallel with skin improvement by steroid therapy. Furthermore, serum aldolase and P-III-P levels decreased after skin improvement. These findings suggest that not only anti-ssDNA Ab levels but also serum aldolase and P-III-P levels are associated with the disease activity. Bone marrow involvement of localized scleroderma has not been reported; however, it may be due to rare MRI examination in affected extremities. Therefore, bone marrow abnormalities should always be considered in patients with localized scleroderma of the extremities. Collectively, not only immunological examinations but also MRI may be useful to evaluate the disease activity and efficiency of the treatment. 


\section{ACKNOWLEDGMENT}

This work was supported by a grant of Research on Intractable Diseases from the Ministry of Health, Labour and Welfare of Japan. 


\section{REFERENCES}

1 Doyle JA, Connolly SM, Winkelmann RK. Cutaneous and subcutaneous inflammatory sclerosis syndromes. Arch Dermatol $1982 ; 118: 886-890$.

2 Takehara K, Sato S. Localized scleroderma is an autoimmune disorder. Rheumatology (Oxford) 2005; 44: 274-279.

3 Emery H. Pediatric scleroderma. Semin Cutan Med Surg 1998; 17: 41-47.

4 Stone J, Franks AJ, Guthrie JA, Johnson MH. Scleroderma "en coup de sabre": pathological evidence of intracerebral inflammation. $J$ Neurol Neurosurg Psychiatry 2001; 70: 382-385.

5 Obermoser G, Pfausler BE, Linder DM, Sepp NT. Scleroderma en coup de sabre with central nervous system and ophthalmologic involvement: treatment of ocular symptoms with interferon gamma. $J$ Am Acad Dermatol 2003; 49: 543-546.

6 Sato S, Ihn H, Soma Y, et al. Antihistone antibodies in patients with localized scleroderma. Arthritis Rheum 1993; 36: 1137-1141.

7 Sato S, Fujimoto M, Hasegawa M, Takehara K. Antiphospholipid antibody in localised scleroderma. Ann Rheum Dis 2003; 62: 771-774. 


\section{FIGURE LEGENDS}

Figure 1. Patient at first visit. (a) Diffuse swelling with the presence of sclerotic plaques on the whole of her right leg. (b) Sclerotic plaques are associated with hypo- and hyperpigmentation.

Figure 2. Biopsy of skin lesion. (hematoxylin-eosin stain) (a) Fibrosis and sclerosis are observed in the whole layer of the dermis. (b) Lymphocytes and plasmacytes infiltrate into the dermis.

Figure 3. T2 weighted MRI findings. (a, b) Images before treatment show high signal intensity lesion of subcutaneous fatty tissue, vastus intermedius, sartorius muscle, femur, tibia, and those bone marrows. (c) Image at eight months after initiating oral corticosteroid therapy shows the reduction of the hyperintense lesion. 
Figure 1.

(a)

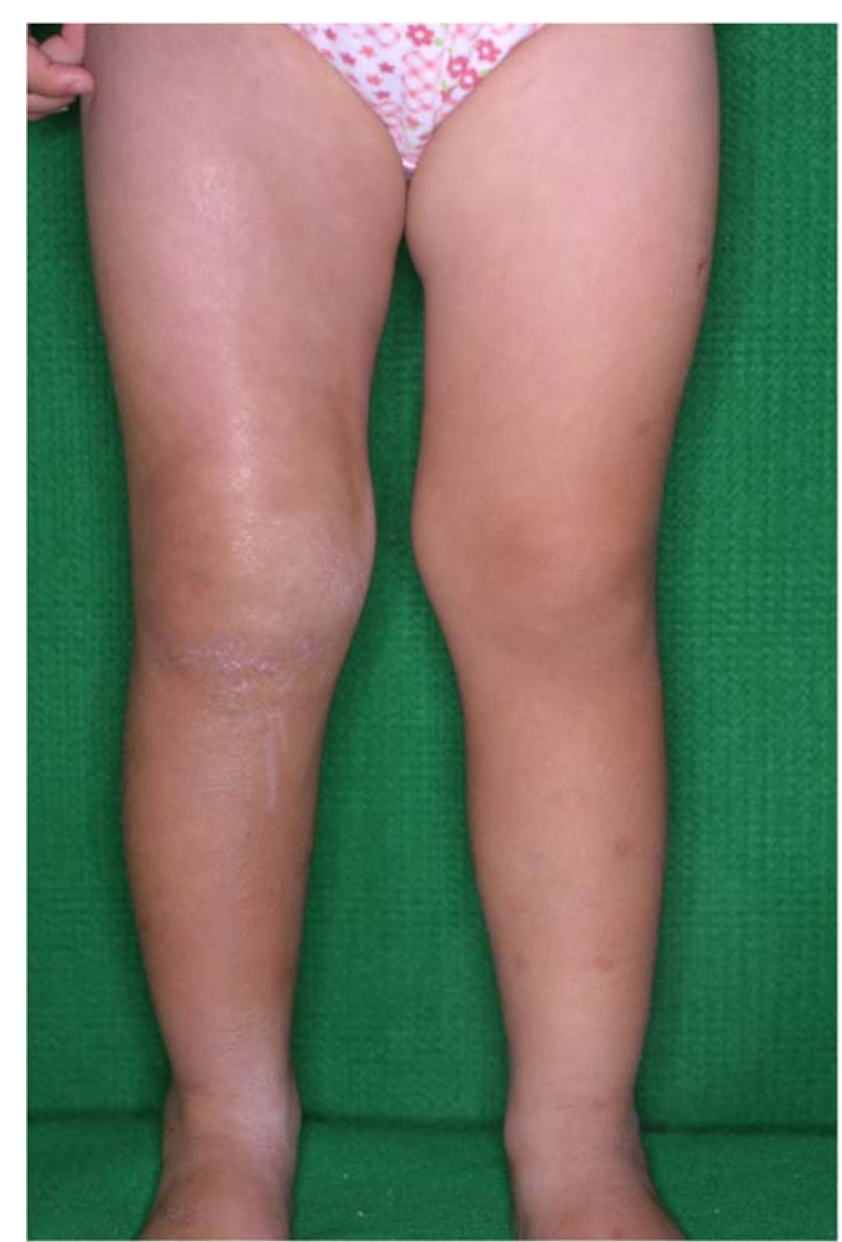

(b)

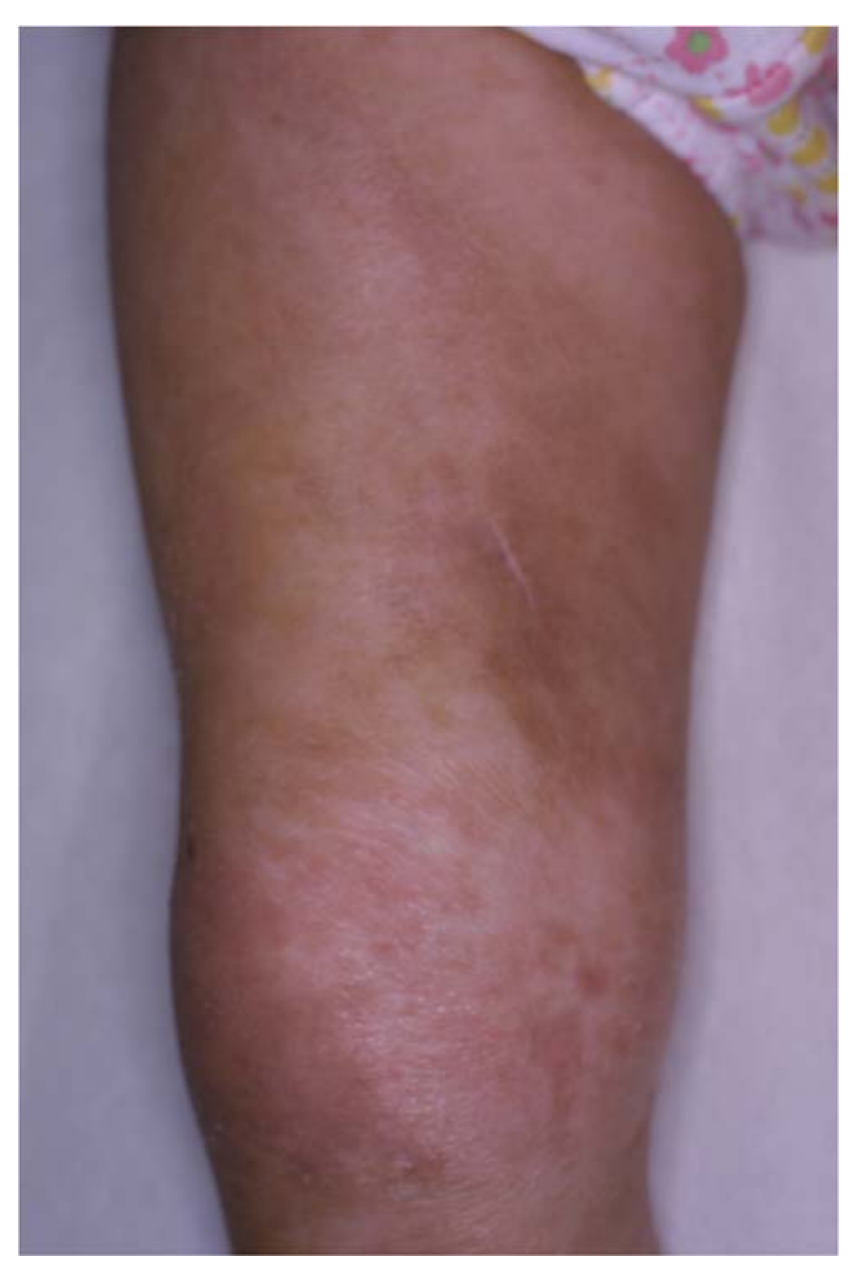


Figure 2.

(a)

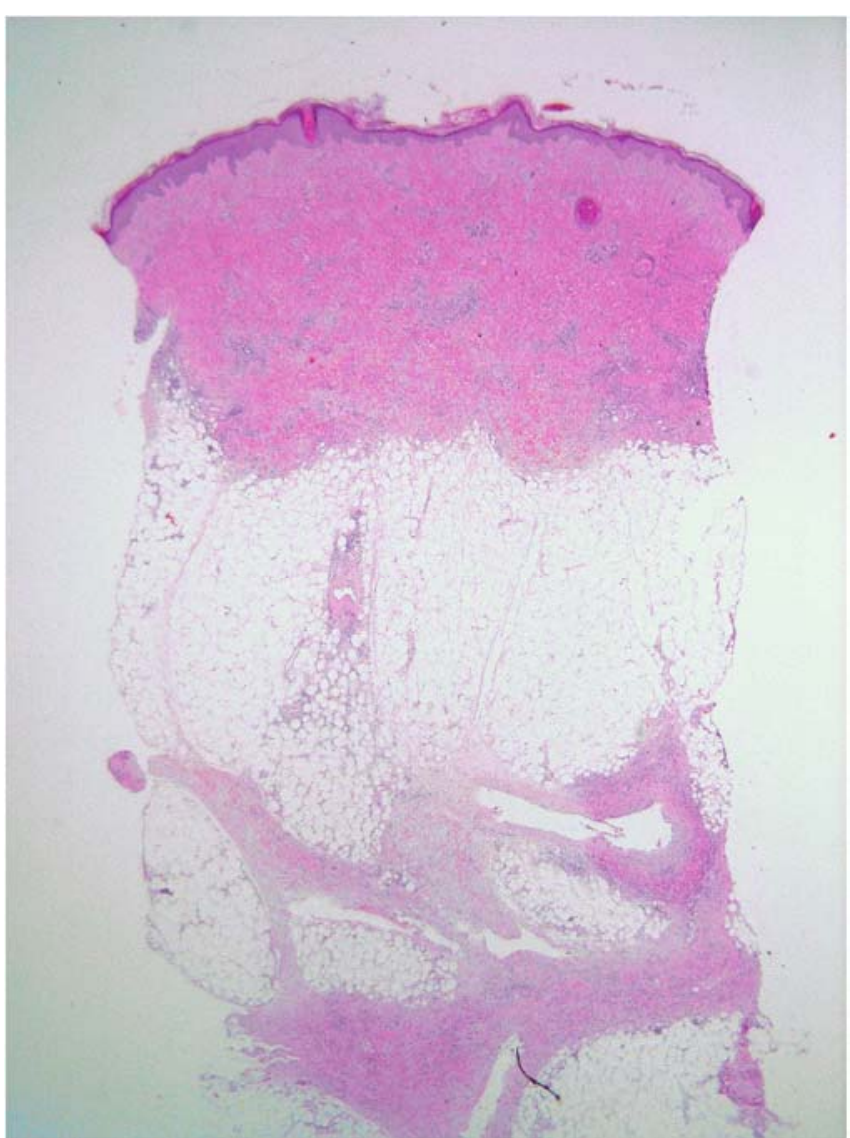

(b)

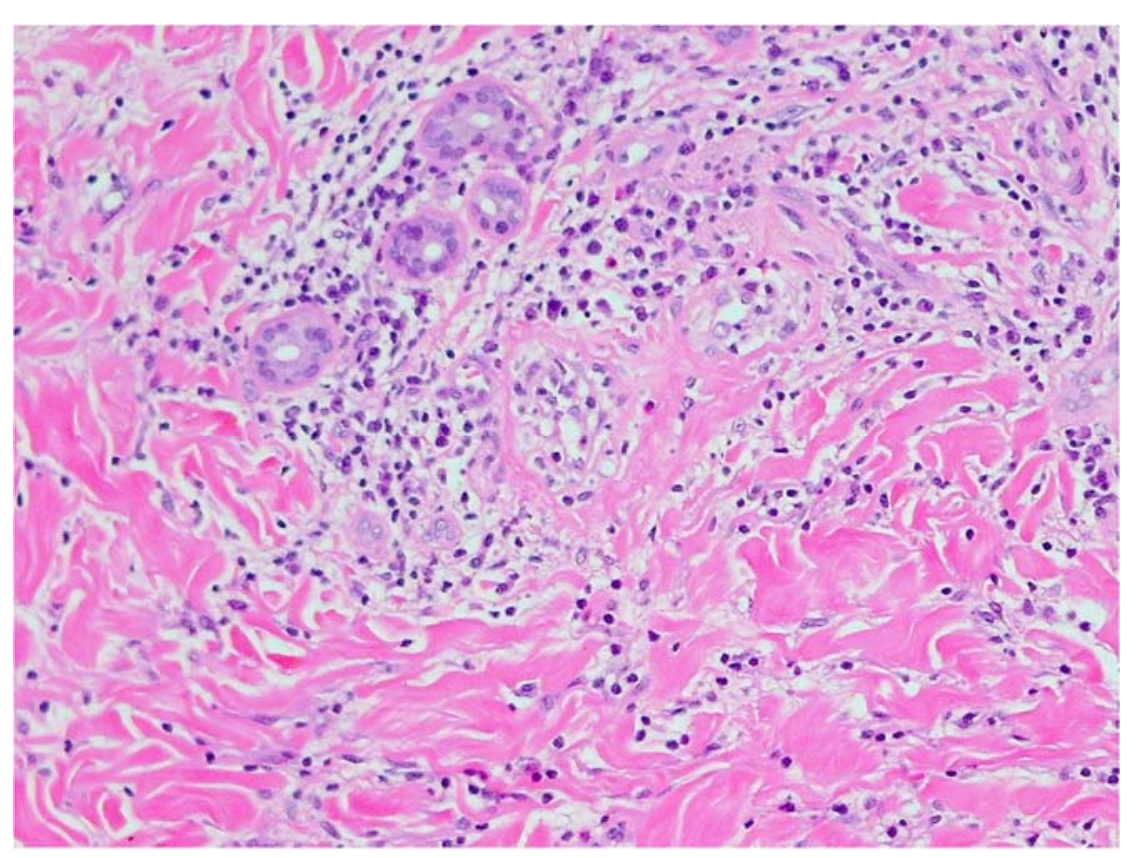


Figure 3.

(a)

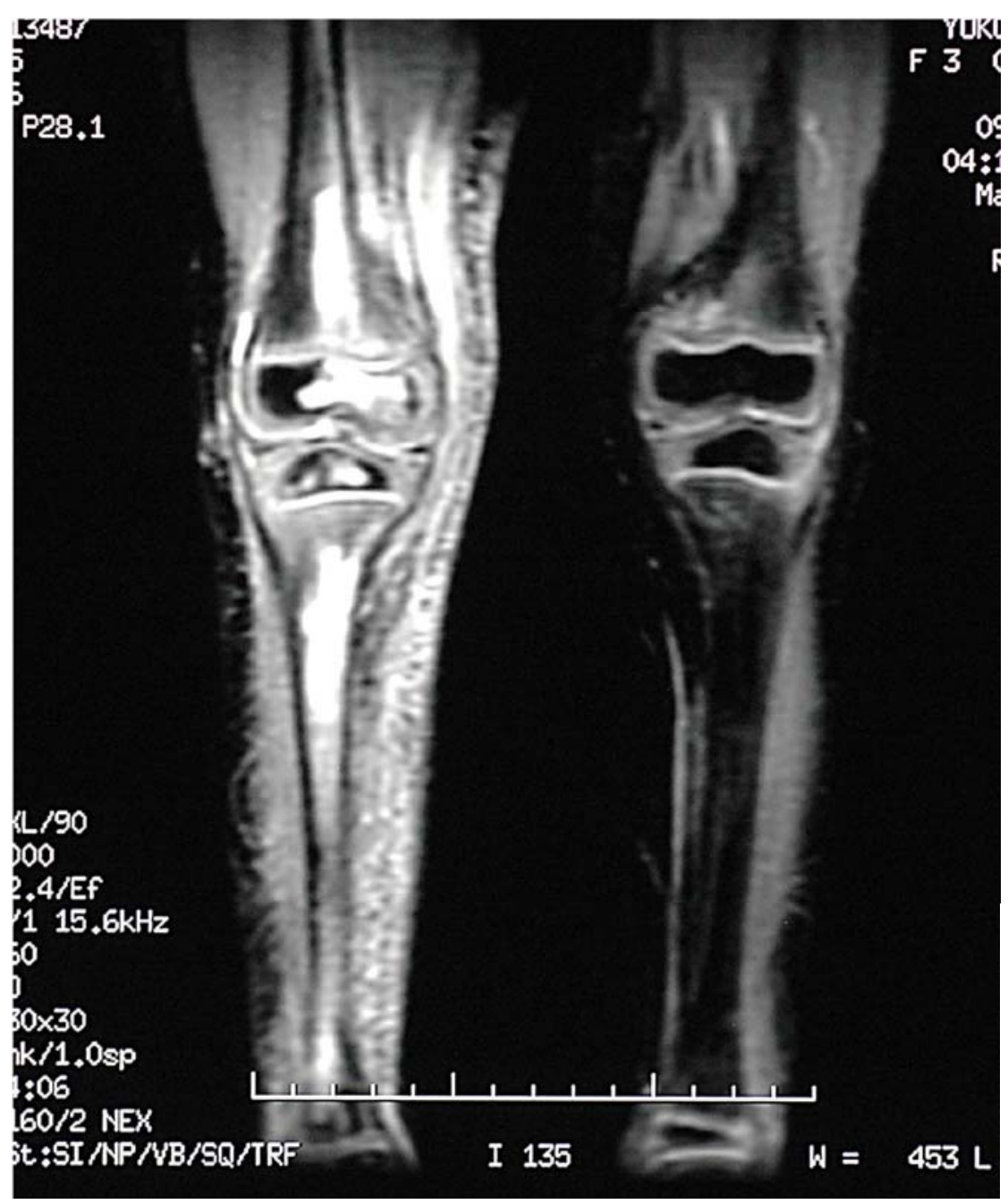

(b)

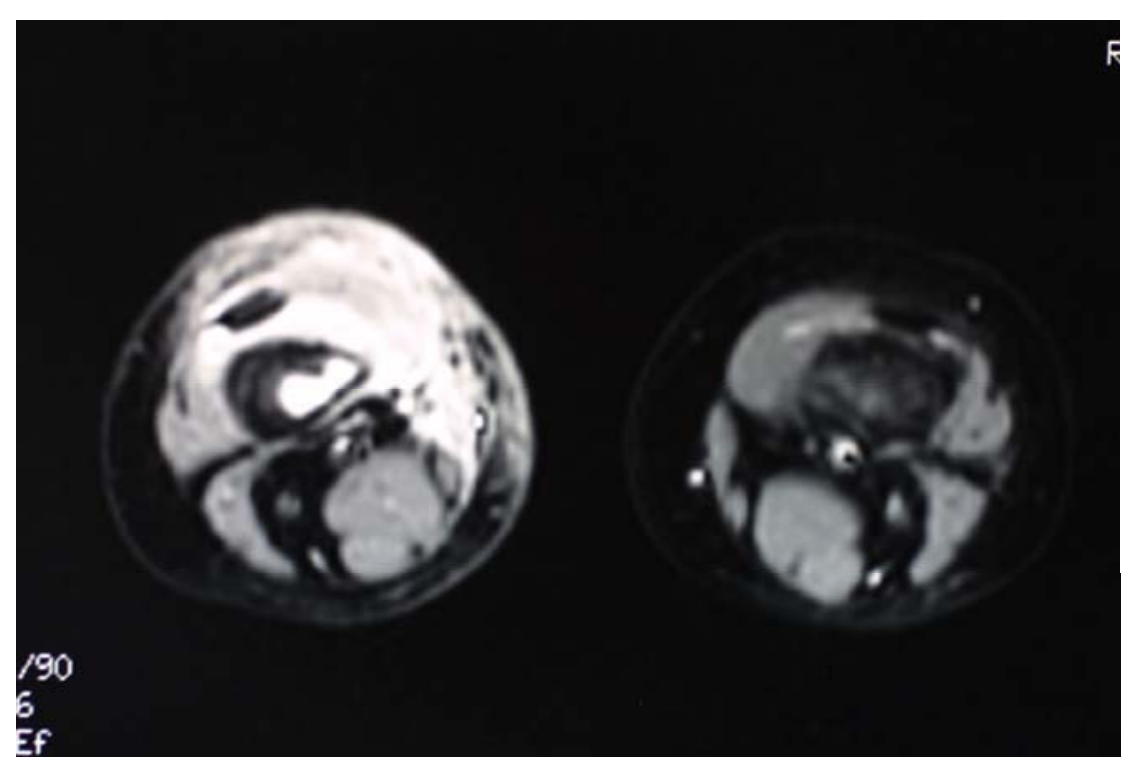

(c)

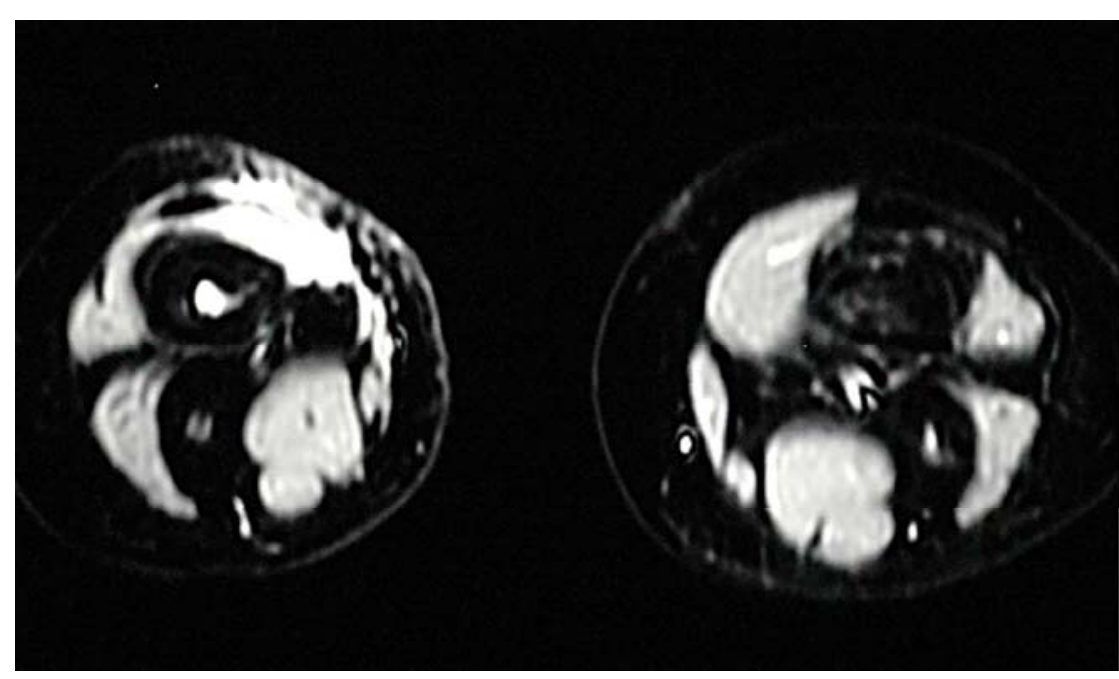

